\title{
Business Of
}

\section{Lifestyle Medicine Practice: Exploring Workable Models}

\begin{abstract}
The practice of lifestyle medicine is different than typical medical practices. Reimbursement systems are strong drivers of practice models. Lifestyle Medicine Solutions (LMS) has developed a primary care lifestyle medicine practice that implements: 1) a new clinical care model, and 2) innovative reimbursement strategies. The key assumptions, care model, reimbursement model and financial partners used by LMS are discussed. To achieve the promise that lifestyle medicine brings for decreasing both the chronic disease burden and rising bealth care costs, new lifestyle medicine delivery models must continue to be explored and implemented. The goal is for the lifestyle medicine choice to be available to each patient and each provider.
\end{abstract}

Keywords: Lifestyle medicine; practice management; reimbursement

ifestyle Medicine Solutions (LMS) is a primary care practice in Southern California that is pursuing a new care delivery model that has a lifestyle medicine (LM) foundation. LMS currently consists of 2 physicians and 1 nurse practitioner. Additional staff include 3 medical assistant/coaches as well as a practice manager/marketer. LMS is a young practice, with its grand opening occurring in May 2015. Although LMS is just now starting to operate in the black, what is being learned has potential applicability to the LM community as a whole.

The premise behind LMS is that a typical physician can practice full-scope LM and actually make a decent living doing so. To achieve this, one must begin with a new approach to not only

\section{Key Assumptions}

The first assumption is that in the current bealth care milieu, Lifestyle Medicine is best practiced when tied to another clinical practice. Although there is a place for a LM-only clinical model, the average patient, health care provider, and health care system do not realize that LM exists. This means that patients are not seeking specialized LM services. If

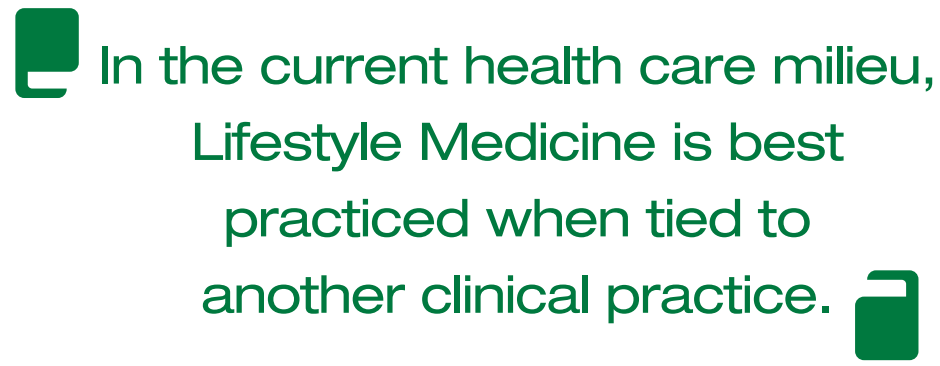

the clinical aspects of health care delivery but also the financial components supporting the services that are provided. Although the clinical aspects are discussed briefly, this article is primarily about the business side of medicine. Other articles have previously been published that discuss the clinical side of LM in further detail. ${ }^{1}$

As the LMS model was being developed, 3 key assumptions were made. These are discussed below, followed by a few pertinent practice details. they learn that such services exist, they may in fact be attracted to them. However, many patients may still be hesitant to make a significant financial investment in such services. Current, successful LM-only clinical practices tend to either cater primarily to the wealthy or be operated by an individual who has written books or in other ways developed a reputation that is hard for the average health care provider to replicate.

If LM is tied to a practice that is already recognized as valuable by the average patient and/or health care system, 
significant access and volume barriers are overcome. In the LMS model, the clinical service foundation is general primary care. In our interactions with health care systems and individual patients, we present ourselves as a primary care practice with a LM flavor. Since primary care is generally recognized and valued, our practice becomes attractive because it is a primary care "plus" practice. Individuals and systems interested in LM are excited to join because we provide a recognized service plus LM as an additional value.

The second important assumption is that Lifestyle Medicine must have a value-based financial reimbursement structure. For LM to really thrive, it is better for the provider to be motivated by a desire to keep a patient healthy, not treat a disease. Typical fee for service reimbursement models reward number of visits or procedures, but not whether a person achieves and maintains health. Although the difference may at times seem subtle, when the size of a provider's take-home pay is directly related to the number of patient visits they provide, their motivation tends to be to provide services in a way that increases the volume of visits. LM struggles in this type of environment.

When a practitioner's income is tied to a capitated, per member per month, or membership-type reimbursement model, the motivation of a provider changes. In these types of systems, the provider, the patient, and the health care system all do better if the patient achieves and maintains health. A provider is then incentivized to spend additional time with the patient, helping him or her to change high-risk health behaviors, and applying the principals of LM. In the LMS model, all our patients are members of our practice. Our reimbursement is a monthly membership fee that is paid for by the patient, the patient's employer, or a government insurance product. We are therefore incentivized to seek out and reverse root causes of disease, not just treat whatever illness may develop.

The third important assumption is that Lifestyle Medicine practices must partner with payers in providing their unique
Table 1.

Disease Risk Factor Assessment.

\begin{tabular}{|c|}
\hline Nutritional status \\
\hline $\begin{array}{l}\text { - Daily vegetable and fruit } \\
\text { servings }\end{array}$ \\
\hline - Liquid calorie intake \\
\hline - Meal and snack schedules \\
\hline - Other details as appropriate \\
\hline Physical activity status \\
\hline - Aerobic, strength, flexibility \\
\hline - Daily living activities \\
\hline Sleep status \\
\hline - Total hours and schedules \\
\hline - Quality of sleep \\
\hline Tobacco use \\
\hline Alcohol use \\
\hline Other drug use \\
\hline
\end{tabular}

Lifestyle Medicine services. Currently, health care services in the United States are predominantly paid for by either the government-primarily through either Medicare or Medicaid products-or by employers-primarily through a variety of commercial insurance products. As health care costs have increased, a growing piece of health care is paid for directly by the patient. Despite this shift, however, health care is still not something that the average consumer approaches with the same type of value-seeking behaviors they may bring to buying food, transportation, housing, or other important purchases.

What this means is that although the typical consumer may feel it makes sense to invest in gym memberships,

supplements, and/or weight loss services, they do not necessarily believe that it is appropriate to individually purchase actual physician-delivered health care services. Since these services have historically been covered by either government or employer-purchased insurance products,
Table 2.

Stress and Social Support Factors.

Marital status

Children (including grandchildren)

Employment status

Living arrangements

- Including who lives in the household

\begin{tabular}{|c|}
\hline Stressors \\
\hline Social support systems \\
\hline Spiritual support systems \\
\hline - Organized religion \\
\hline - Sense of purpose \\
\hline $\begin{array}{l}\text { - Relationship with God or } \\
\text { equivalent }\end{array}$ \\
\hline
\end{tabular}

there remain deeply held beliefs that this should continue to be the case. There are exceptions to this rule. Certain

individuals—usually either the wealthy, or people fed up with a chronic disease they feel has not been properly addressed by the typical health care system-are happy to pay for additional LM services. Most, however, are not. To achieve widespread adoption, physician-delivered LM services must therefore be primarily purchased by the payer rather than the patient.

\section{The LMS Care Model}

In the LMS model, understanding the patient and being fully present to their needs is the key objective. To understand the patient, a comprehensive health risk assessment, called the patient profile, is implemented for each patient. Some details of this are described in Tables 1 and 2. Because it is a membership practice, LMS does not wait for patients to schedule a visit. Each patient is tracked with a connectivity index that measures and designates a follow-up contact method and connection time. Connectivity can occur in person, or via 
phone, email, or text. Patients choose their health goals for the year. The connectivity index is then used to help them achieve those goals.

The LMS model provides care through a team-based approach. Medical assistants have additional training as a health navigator and coach. Each patient is assigned to both a provider and a medical assistant/coach. Both the provider and the medical assistant/coach interact with the patient in the office, via phone, via email, and through text messaging. Additional communitybuilding activities also occur regularly within the practice. Among other things, these include group visits, cooking classes, health fairs, and "walk with the doc" events.

Although the LMS model has only 3 job descriptions-provider, medical assistant/ coach, and practice manager-multiple contract providers are also involved in the practice. This includes a dietician, a personal trainer, a counselor/

psychologist, a life coach, and a certified functional medicine provider. A variety of community practitioners and specialists who understand and value the LMS approach to care delivery have also been identified and are used as primary referral sources.

\section{The LMS Membership Levels}

The LMS financial model allows members to join at multiple levels. The lowest membership level is free. At this level members receive the LMS weekly newsletter, invitations to participate in certain community-building events, and discounts for additional LMS offerings. Patients who want to engage in the practice more actively, but who self-identify as either having lower health care needs or strong commercial preferred provider insurance, can join the practice at $\$ 15$ per month. At this level they can participate in group visits and receive discounts on other provider services.

The next level of membership is available at $\$ 60$ per month. At this level the patient receives the annual assessment and health profile, along with limited follow-up visits with the physician or nurse practitioner. Additional services are at a significant discount

Two other premium levels of membership also exist. For $\$ 120$ per month, patients achieve all of the components of the lower membership fees, plus unlimited interactions with the provider. These may again be via text, email, phone, or in person, and may occur in group settings or be facilitated by the medical assistant/coach. For $\$ 180$ per month, patients not only receive all the benefits of the less expensive membership plans, they are also eligible for additional packages. These include a free intensive therapeutic lifestyle (ITLC) program, visits with the dietician or personal trainer, further assessments with the functional medicine provider, and other package options customized for the individual patient.

\section{The LMS Financial Partners}

LMS has partnered with several organizations that support components of the membership fee. LMS has also developed productive relationships with a number of Medicare Advantage (MA) programs. From an MA program perspective, they are paying LMS a capitated, per member per month fee, and getting good value for their dollar. From the LMS perspective, the MA programs are paying the monthly membership fee. Contractually, the MA programs begin their reimbursement somewhat below the $\$ 120$ per month membership fee. Risk adjustment factors and other contractual reimbursement components can expand the per member per month reimbursement total to nearer the $\$ 180$ per month membership fee.

LMS has also partnered with Liberty Direct (LD), which is a health care expense-sharing program. Although it is a cost-sharing program, not health insurance, LD is approved by the Affordable Care Act as a health insurance equivalent, thereby eliminating any penalties that would otherwise accrue for those not carrying health insurance. LD will pay for the $\$ 60$ per month LMS membership fee.

Since both the MA programs and LD are driven by value-based reimbursement models, there are obvious synergies between them and LMS. They thrive if month-to-month and year-to-year overall health care costs are decreased, and they see LM approaches helping achieve these health care cost savings. The LMS partnerships with these organizations helps LMS continue their focus on achieving health, and steer away from the disease care approach that tends to drive most current health care practices.

LMS believes that every patient, when faced with a chronic disease, should be given the LM choice. Too often, right now, they are not. LMS also believes that every provider who wants to provide LM-based health care services should be able to do so while accomplishing reimbursement that is in the same range as similar non-LM-based providers. The LMS approach is one model that appears to be achieving this. Now is the time to be exploring and adopting new care delivery models that are LM based. Others are invited to join as the dialogue around reversing the chronic disease epidemic through LM is expanded.AJLM

\section{Reference}

1. Dysinger WS. Lifestyle medicine: an overview. Prim Care Rep. 2010;16(5):1-12. 